\title{
Do we need day-1 postoperative follow-up after cataract surgery?
}

\author{
Andrzej Grzybowski ${ }^{1,2} \cdot$ Piotr Kanclerz ${ }^{3}$ \\ Received: 30 March 2018 / Revised: 21 November 2018 / Accepted: 4 December 2018 / Published online: 19 December 2018 \\ (C) The Author(s) 2018
}

\begin{abstract}
Purpose The aim of our study was to evaluate the current nature and frequency of complications present on the first postoperative day (POD1) and to verify whether the completion of a follow-up visit at this time is justified after standard phacoemulsification cataract surgery (PCS).

Methods We used the PubMed literature database to identify relevant studies using the following keywords: postoperative, follow-up visit, complications, outcome, intraocular pressure, IOP, intraocular pressure spikes, IOP spikes, wound leakage, wound dehiscence, intraocular lens, IOL, dislocation, exchange, phacoemulsification, cataract surgery, and cataract extraction. Results We collected and analyzed 45 articles published between 1994 and 2017. The most common complications after PCS include corneal edema, postoperative uveitis, intraocular pressure (IOP) elevation, cystoid macular edema, and posterior capsule opacification. The IOP typically peaks at 3 to $7 \mathrm{~h}$ after surgery; however, none of the assessed treatment regimens were sufficient to protect glaucomatous eyes from IOP spikes. The majority of postoperative complications do not require early surgical intervention. Alternatives to POD1 follow-up after PCS include a nurse-administered telephone questionnaire, shared care with non-ophthalmologists, and seeing the patients at a low threshold in cases of complaints.

Conclusions The current literature does not support the concept of a POD1 follow-up after uneventful PCS in patients without posterior synechiae or chronic/recurrent uveitis and operated on by experienced surgeons. When eliminating the POD1, visit individuals should receive topically a potent steroid (preferably prednisolone or dexamethasone). Applying a combination of topical dorzolamide/timolol and brinzolamide postoperatively in patients with glaucoma would be recommended, particularly in eyes with preexisting optic nerve damage. Eliminating the routine POD1 follow-up could result in significant health care savings without an increased risk to the patient.
\end{abstract}

Keywords Cataract surgery $\cdot$ Follow-up $\cdot$ Complications $\cdot$ Ocular hypertension $\cdot$ Patient safety $\cdot$ Phacoemulsification

\section{Introduction}

The consistent and ongoing increase in health care costs necessitates a verification of current practice patterns and the elimination of redundant and low-value services. The problem is not trivial as the impact of superfluous tests and procedures in the United States (US) was estimated be as high as $30 \%$ of all health care spending, with just non-recommended activities

Andrzej Grzybowski

ae.grzybowski@gmail.com

1 Department of Ophthalmology, University of Warmia and Mazury, Olsztyn, Poland

2 Foundation for Ophthalmology Development, Institute for Research in Ophthalmology, Gorczyczewskiego 2/3, 60-554 Poznan, Poland

3 Hygeia Clinic, Gdańsk, Poland in primary care accounting for an annual cost of $\$ 6.76$ billion [1]. Up to $97.1 \%$ of clinicians believe that the current frequency of unnecessary tests and procedures is a serious problem [2].

The timing of postoperative examinations should be adjusted to ensure the expeditious recognition and management of complications in order to optimize the final outcome of surgery. Several practice patterns exist worldwide. The American Academy of Ophthalmology recommends a first-day postoperative (POD1) visit should be done in functionally monocular patients, following intraoperative complications, or in those at a high risk of immediate postoperative complications such as intraocular pressure (IOP) spike. In patients without these risks the follow-up visit should be scheduled within $48 \mathrm{~h}$ [3]. According to the Royal College of Ophthalmologists, however, POD1 review is no longer in widespread use in the United Kingdom (UK). Such an examination is recommended only in complicated surgeries, in eyes with co-existing diseases (e.g., 
glaucoma, uveitis), or in patients with only one eye [4]. Upon discharge, comprehensive information to identify potential complications should be provided, with a postoperative appointment date confirmed and the required medications dispensed.

The Cochrane Database for Systematic Reviews found cost savings associated with same-day discharge (SDD) cataract surgery versus in-patient cataract surgery; however, the evidence regarding postoperative complications was inconclusive because the effect estimates were imprecise [5]. The necessity for a POD1 follow-up in routine phacoemulsification was questioned more than 20 years ago [6,7], and more recently in vitreoretinal surgery [8]. The aim of the present study was to evaluate the current nature and frequency of complications present at POD1 in small-incision phacoemulsification cataract surgery (PCS) and to determine which individuals might require routine review at this time. Uneventful cataract surgery was defined as a procedure with no intraoperative complications (e.g., posterior capsule rupture, vitreous loss, lost nucleus, and zonular dehiscence).

\section{Materials and methods}

PubMed and Medline were used to identify relevant studies and the search was focused on articles evaluating the nature and frequency of complications after PCS up to Dec 31, 2017. The following keywords were used in various combinations: postoperative, follow-up, visit, complications, outcome, intraocular pressure, spikes, wound leakage, wound dehiscence, intraocular lens, IOL, dislocation, exchange, phacoemulsification, cataract surgery, and cataract extraction. The search identified 1167 unique articles and only English language articles were selected. Articles cited in the reference lists of other publications were also considered as a potential source of references. Studies were critically reviewed to create an overview and guidance for further search. No attempts to discover unpublished data were made. Articles providing original research and case reports were included. Conclusions from review articles were taken into consideration if relevant to the topic.

\section{Results}

\section{Frequency of postoperative complications}

Via our literature review, we collected and analyzed 44 articles published between 1994 and 2017. The prevalence of postoperative complications in some of the largest studies on cataract surgery is presented in Table 1. According to the Cataract National Dataset of the UK, the most common postoperative complications were "corneal edema, striae, and Descemet's
Table 1 Prevalence of postoperative complications in selected cataract surgery studies

\begin{tabular}{llll}
\hline & $\begin{array}{l}\text { Jaycock et al. 2009 } \\
{[9](n=16,731)}\end{array}$ & $\begin{array}{l}\text { Syed et al 2015 } \\
{[10](n=20,070)}\end{array}$ & $\begin{array}{l}\text { Greenberg et al. 2011 } \\
{[11](n=45,082)}\end{array}$ \\
\hline Corneal edema/striae/Descemet folds & $5.18 \%$ & $0.59 \%$ & N/A \\
Postoperative uveitis & $3.29 \%$ & $0.24 \%$ & N/A \\
Raised IOP $>21 \mathrm{mmHg})$ & $2.57 \%$ & $0.31 \%$ & N/A \\
Cystoid macular edema & $1.62 \%$ & $0.22 \%$ & $3.3 \%$ \\
Posterior capsule opacification & $1.22 \%$ & $0.34 \%$ & $4.2 \%$ \\
Iris prolapse/iris to wound & $0.16 \%$ & $0.04 \%$ & N/A \\
Retained soft lens matter & $0.45 \%$ & $0.19 \%$ & $1.7 \%$ \\
Vitreous to section & $0.39 \%$ & $0.09 \%$ & N/A \\
Vitreous in the AC & $0.17 \%$ & N/A & N/A \\
IOL decentration/dislocation/exchange & $0.22 \%$ & N/A & $0.9 \%$ \\
Wound leak & $0.14 \%$ & $0.02 \%$ & N/A \\
Choroidal effusion/hemorrhage & $0.13 \%$ & N/A & $0.1 \%$ \\
Hyphema & $0.07 \%$ & N/A & $0.2 \%$ \\
Hypopyon/endophthalmitis & N/A & $0.04 \%$ & $0.2 \%$ \\
TASS & N/A & $0.1 \%$ & N/A \\
Ptosis & N/A & $0.01 \%$ & N/A \\
Retinal tear or detachment & N/A & $0.03 \%$ & $1.0 \%$ \\
Vitreous hemorrhage & N/A & $0.01 \%$ & $0.4 \%$ \\
Hypotony & N/A & N/A & $0.1 \%$ \\
\hline
\end{tabular}

Complications with the prevalence rate of $1 \%$ or more are presented in italics. $A C$, anterior chamber; $I O L$, intraocular lens; IOP, intraocular pressure; N/A, not available; TASS, toxic anterior segment syndrome 
membrane folds" ( $5.18 \%$ cases), followed by postoperative uveitis $(3.29 \%)$, IOP rise above $21 \mathrm{mmHg}(2.57 \%)$, cystoid macular edema (1.62\%), and posterior capsule opacification (1.22\%) [9]. The UK Specialist Hospital benchmark presented significantly lower complication rates in 20,070 cataract surgeries, with none of the formerly mentioned disorders reaching a prevalence of $1 \%$ [10]. In the US Veterans National Patient Care Database, the most common postoperative complication in 45,082 cataract surgeries were posterior capsule opacification and cystoid macular edema [11].

\section{IOP spikes}

In the study by McKellar and Elder on the POD1, complications were observed in $10 \%$ of 1000 surgeries, while heightened IOP represented $88 \%$ of all complications [12]. In more recent studies, a rise in IOP over $21 \mathrm{mmHg}$ was observed in 0.31 to $2.57 \%$ of cases $[9,10]$. In high-risk patients, early postoperative IOP increase above $28 \mathrm{mmHg}$ was reported in up to $46.4 \%$ of high-risk patients [9]. The risk factors for IOP spikes following PCS include residual viscoelastic material [13-15], resident-performed surgery [16-18], glaucoma [19, 20], exfoliation syndrome [21], axial length of more than $25 \mathrm{~mm}$ [22], tamsulosin intake [23], and topical steroid application in steroid responders [24]. Postoperative anterior chamber inflammation might also result in an early IOP increase [25]. In these cases, an effective way of controlling the postoperative IOP is to individualize the frequency of topical steroid administration.

The IOP typically peaks at 3 to $7 \mathrm{~h}$ postoperatively and remains increased during the first $24 \mathrm{~h}$ after surgery. Although the increase is usually transient and does not influence the long-term quality of vision, IOP spikes are potentially more dangerous in eyes with preexisting optic nerve damage, such as in patients with glaucoma or atherosclerosis-related ischemia. Occasionally, a patient with a pressure of 40 to $50 \mathrm{mmHg}$ will experience pain and perhaps nausea, resulting in dissatisfaction with the surgery or a phone call to the surgeon in the middle of the night.

Several topical IOP-lowering agents have been evaluated, but none to dates have completely prevented the occurrence of IOP spikes. Kandarakis et al. found that a single dose of brimonidine tartrate $0.2 \%$ after cataract surgery reduced IOP elevation, but was not sufficient to protect glaucomatous eye from IOP spikes [26]. In a separate study, the topical administration of $0.5 \%$ timolol maleate at the end of surgery eliminated IOP spikes to over $30 \mathrm{mmHg}$ and reduced the frequency of IOP rise to more than $25 \mathrm{mmHg}$ to $14 \%$ in the glaucoma group and $5 \%$ in the exfoliation syndrome group, respectively [27]. Furthermore, Takmaz et al. noted that topical application of bimatoprost in eyes with exfoliation syndrome is effective in preventing IOP spikes $\geq 30 \mathrm{mmHg}$ in the early postoperative period [28]. It might be concluded that postoperative application of a combination of dorzolamide/timolol and brinzolamide topically in high-risk patients with raised IOP or preexisting optic nerve damage may be appropriate. A POD1 follow-up visit might be questioned, even in glaucoma patients, as any IOP elevation that will occur typically reaches its highest peak at just a few hours postoperatively.

\section{Wound dehiscence}

The transition to small-incision phacoemulsification resulted in a decrease in the rate of wound dehiscence from 2.8\% [29] to $0.02-1.1 \%[10,30,31]$. With that, it is possible to avoid complications from sutures [32]. Currently, surgical revision is required infrequently; for example, in a study by Zaidi et al. of the 11 of 1000 consecutive patients undergoing PCS with wound dehiscence, only one eye required a reoperation with formal wound revision [30]. Tamsulosin intake might increase the probability of wound dehiscence, with a relative risk of 3.81 times that seen in the control group [33]. As patients taking tamsulosin manifest a pronounced risk for wound dehiscence, it was suggested that this group might require suturing of the corneal incision [33].

Intraoperative wound leakage is usually observed at the side-port incisions and simply requires additional hydratation [34]. Poor wound apposition is believed to result in postoperative hypotony, predisposing the affected individual to an intraocular infection. Interestingly, in a study by Shingleton, neither paracentesis nor keratome incision leakage was noted by fluorescein strip testing at the completion of surgery in eyes with hypotony, which was defined as an IOP lower than $10 \mathrm{mmHg}$ at $30 \mathrm{~min}$ postoperatively [35]. It was concluded that early hypotony may be a result of a disequilibrium between postoperative aqueous production and outflow, rather than incisional leakage. With that said, suturing did not affect the incidence of postoperative hypotony in this study.

\section{Inflammatory response after surgery}

It is postulated that physical trauma related to surgical manipulations within the anterior chamber may induce an inflammatory response. The release of arachidonic acid from uveal tissue with the production of leukotrienes (via the lipoxygenase pathway) or prostaglandins (via the cyclooxygenase pathway) results in the subsequent disruption of the blood-aqueous barrier. Most authors agree that all eyes undergoing PCS should be free of any cell/flare for a minimum of 3 months before surgery. Patients with recurrent or chronic uveitis usually may undergo surgery during a "window of opportunity" when the inflammation seems to be better-controlled [36]. In this case, preoperative or postoperative oral prednisolone, intraoperative treatment with intravenous methylprednisolone, and/or immunosuppressive treatment might be recommended. A limitation in assessing the risk of postoperative uveitis after PCS in a general population is that 
some of the analyzed cohort studies did not present the postoperative anti-inflammatory regimen [9-11], or do not report the percentage of patients with preoperative uveitis/ posterior synechiae [10]. In the study by Jaycock et al., uveitis/posterior synechiae were present in $0.96 \%$ of individuals preoperatively, and $3.29 \%$ of patients developed postoperative uveitis [9], while Syed et al. reported the prevalence of postoperative uveitis as only $0.24 \%$ [10]. It might be concluded that when eliminating the POD1 visit, the patients should receive a prompt antiinflammatory treatment, including a potent steroid (preferably prednisolone or dexamethasone). Individuals with preoperative uveitis or posterior synechiae might require a periocular steroid injection, or preferably a POD1 visit.

Toxic anterior segment syndrome (TASS) is a sterile postoperative inflammatory reaction that usually presents at 12 to $48 \mathrm{~h}$ after surgery. TASS can easily be misdiagnosed since it mimics endophthalmitis with occasional reports of early vitrectomy as an unnecessary treatment. B-scan ultrasonography is useful in differential diagnosis, as vitreous opacities are usually not present in TASS. In a single institution study, TASS occurred in 60 eyes $(0.22 \%)$ of a sample of 26,408 consecutive cataract surgeries [37]. Ozcelik et al. reported an incidence of $0.8 \%$ among 1742 surgeries [38]. The application of intensive topical antiinflammatory treatment usually results in good visual outcomes. It might be presumed that TASS, as it results in a marked decrease in vision, eye redness, and occasionally pain and photophobia, can be easily noted by the patient.

The postsurgical inflammatory reaction might also result in cystoid macular edema. In the study by Greenberg et al., cystoid macular edema was the second most common postoperative complication of cataract surgery with the prevalence of 3.3\% [11]. It should be underlined that cystoid macular edema usually develops between 1 and 6 weeks after surgery [39]. Moreover, some retinal diseases diagnosed preoperatively, including neovascular age-related macular degeneration and diabetic retinopathy, might justify additional perioperative therapy, including anti-vascular endothelial growth factor or/and non-steroidal antiinflammatory drugs (NSAID) treatment. Setting the treatment regimen is commonly based on a scrupulous preoperative examination rather than on the POD1 visit results. However, postoperative dosing of a topical steroid might be related to the degree of postoperative inflammation.

\section{IOL dislocation}

In a study by Greenberg et al., $0.9 \%$ of patients presented with IOL dislocation or underwent IOL exchange in a 90-day postoperative period [11]. Early IOL dislocation is usually related to improper fixation of the IOL within the capsular bag, issues with asymmetric IOL fixation, or the presence of an incomplete or torn capsulorhexis [40]. In recent years, the incidence of early IOL dislocations has decreased as a result of using a continuous curvilinear capsulorhexis and improved phacoemulsification techniques. Currently, most of the postoperative IOL dislocations present spontaneous in-the-bag dislocations and take place in the late postoperative period (i.e., more than 3 months after surgery) [41].

In a survey by Mamalis et al., IOL dislocation/decentration was found to be the most common reason for IOL explantation [42]. One of the root causes of such was inadequate zonular support for the capsular bag-IOL complex. A review by Vazquez-Pereiro et al. revealed that patients with pseudoexfoliation confer an increased risk for both early and late IOL dislocation [43]. Other reasons for IOL exchange include incorrect IOL power, IOL opacification, or a failure by the patient to adapt to multifocal IOLs [44]. With the formerly mentioned indications, IOL dislocation might require an immediate intervention.

\section{Intraoperative complications}

As previously stated, the American Academy of Ophthalmology recommends a follow-up visit be completed within $24 \mathrm{~h}$ after surgery in the case of patients with intraoperative complications [3] The most common intraoperative complication in cataract surgery is posterior capsule rent (PCR), with or without vitreous loss; its prevalence being estimated as 1.92 to $3.5 \%[9,11]$. PCR might result in several postoperative conditions such as vitreous in the anterior chamber or vitreous strand towards the section. If the vitreous in the anterior chamber is not cleared, it can lead to endothelial damage and corneal edema. Increased IOP can be a result of trabecular meshwork obstruction by retained ophthalmic viscoelastic device, vitreous in the anterior chamber, inflammatory cells, or pigment dispersion related to excessive intraocular manipulations. Posterior capsule rupture might also lead to uveitis, cystoid macular edema, or retinal detachment [45]. Similarly, the retained soft lens matter might result in increased IOP, uveitis, corneal edema, cystoid macular edema, or retinal detachment.

Other less frequent intraoperative complications include iris prolapse, vitreous hemorrhage or hyphema, iridodialysis, and suprachoroidal hemorrhage. Although the incidence of intraoperative complications in cataract surgery is relatively low, there is no evidence to eliminate a 24 -h postoperative follow-up visit after a complicated surgery even in highvolume practices.

\section{Studies evaluating the necessity for POD1 follow-up visit and alternative options}

A systematic review conducted to assess the value of early postoperative examinations was completed by Kessel et al. [46]. No significant degree of better safety was gained by reviewing the patients on POD1, and deferment of the postoperative review was suggested in low-risk patients. A closer follow-up was recommended in patients with glaucoma in 
order to evaluate postoperative IOP spikes, in eyes with intraoperative complications, or in those patients operated on by less-experienced surgeons. Tan et al. suggested that the POD1 hospital visit may be safely managed by way of a nurseadministered telephone questionnaire with patient contentment achieved and the liberation of clinic resources ensured [47]. In their study, only one of 238 patients reported a poor general condition, blurred vision with pain, and was asked to return for a clinic review on POD1. Tufail and associates compared the complications seen with SDD surgery (with a review occurring at 4 to $6 \mathrm{~h}$ postoperatively) with those seen with in-patient surgery (with a POD1 review) and found that the most common complications in both groups were IOP rise, corneal edema, and wound leaks [7]. Only one patient in each group, with an iris prolapse, required attending to the department. It was concluded that there were no additional risks related to SDD surgery without a POD1 review.

Allan et al. reported a low intervention rate of $2.8 \%$ in 1652 routine follow-up visits [48]. Additionally, $7.3 \%$ of patients made unscheduled emergency service visits with an intervention rate of $50 \%$. It was concluded that alternatives to conventional postoperative review, including shared care with nonophthalmologists and improved perioperative patient education with an open channel for self-referral, should be considered. The Royal College of Ophthalmology recommends that the postoperative follow-up examinations should be carried out by an informed nurse, optometrist, or ophthalmologist [4].

With that in mind, Eloranta et al. suggested that a postoperative check-up visit might not be required at all in the majority of cases [49]. In the year 2006, a follow-up visit was advised to occur 1 month after surgery, while, in 2009, patients were informed that such an appointment is not necessary. Contacting the department was advised if they experienced pain, vision deterioration, or ocular discharge, and only those patients with intraoperative complications or comorbidities influencing postoperative recovery were selected for a follow-up visit. In the postoperative period, $4.2 \%$ of patients in 2006 and $3.9 \%$ of patients in 2009 contacted the hospital because of symptoms. Referral was necessary in only $0.5 \%$ of patients in the 2006 cohort, and $0.3 \%$ of patients in the 2009 cohort, while a surgical or medical intervention was needed by only one third of these individuals. It was concluded that the lack of a 1-month check-up did not influence patient's safety; however, in cases with intraoperative problems, comorbidities influencing recovery, or postoperative symptoms, the patients should be seen at a low threshold.

\section{Socioeconomic considerations and non-quantifiable benefits of POD1 visit}

The annual cost of postoperative visits in cataract surgery in the US is estimated as $\$ 0.8$ billion. Usually, the service is reimbursed by the insurance carrier and is not an out-of- pocket expense for the patient. In some countries, the postop care is bundled with the surgical fee or might be required by the payer. Nevertheless, even in these settings, human work is expensive and the freed up resources could be used to manage other health problems.

Commonly, cataract surgeries are performed as SDD surgery. The direct non-medical costs (e.g., transport, accommodation, and meals for patients and possibly their families) might constitute $49 \%$ of the total direct cost of cataract surgery, particularly in developing countries [50]. In developing countries, patients may travel further to available or better facilities. As such, the current trend is that the postoperative follow-up is done by ophthalmologists. If there is no complication, the standard postoperative routine may be started. The patient must be informed of any complication. The surgeon then must decide whether to follow the patient or refer to a subspecialist or local practitioner. The final follow-up is done after 6 weeks with the optometrist for possible refraction and spectacle prescription. This practice saves many patients from renting hotel rooms, or at least doing so for a longer period. In developed countries where patients largely do not have to travel far and stay in hotels for operations, doing away with most POD1 visits would produce less patient savings. It might be assumed that applying these recommendation worldwide may not be suitable or possible. In many European countries, including Poland, a POD1 visit is advised by the national ophthalmic society and required by the payer, i.e., the National Health Service. We believe that this study could begin a discussion about the requirement of POD1 visit in all cases, and could eventually result in giving a choice to the surgeon in charge.

In future research, it would be beneficial to analyze the psychosocial aspect of postoperative visits with regard to patients and their relatives. Patients may potentially feel more satisfied after being seen and having their treatment regimen planned by the operating surgeon on the POD1. Surgeons may also feel more comfortable after seeing their patients postoperatively and only plan the additional treatment or follow-up visits according to the clinical findings seen on POD1. With that, even if one severe complication in 100 or 1000 cases is detected, one might believe that this makes such a visit worthwhile. The great majority of patients will be happy to hear that their eye is healing well and to have their vision and IOP measured, especially if their vision is good. If the vision is poor, the patients and caregivers need to know why (e.g., due to corneal edema or any other reason) and what the final prognosis is. This also is an opportunity to review the use of postoperative drops, which elderly patients in particular may find confusing.

\section{Conclusions}

The current literature does not support the concept of POD1 follow-up visit after uneventful cataract surgery. Although the 
incidence of intraoperative complications in cataract surgery is relatively low, there is no evidence to eliminate a $24-\mathrm{h}$ postoperative follow-up after a complicated surgery, operated on by less-experienced surgeons, or in patients with chronic/ recurrent uveitis or posterior synechiae. It is advised that patients experiencing any issues should be seen in the postoperative period at a low threshold. Applying a combination of topical dorzolamide/timolol and brinzolamide postoperatively in patients with glaucoma may be recommended, especially in eyes with preexisting optic nerve damage. Eliminating the routine POD1 follow-up could result in significant health care savings without an increased risk to the patient.

\section{Compliance with ethical standards}

Conflict of interest Dr. Grzybowski reports grants, personal fees and non-financial support from Bayer, non-financial support from Novartis, non-financial support from Alcon, personal fees and non-financial support from Valeant, and non-financial support from Santen outside the submitted work. Dr. Kanclerz reports non-financial support from Visim. No conflicting relationship exists for any author.

Ethical approval This article does not contain any studies with human participants performed by any of the authors.

Abbreviations IOL, intraocular lens; IOP, intraocular pressure; NSAIDS, non-steroidal anti-inflammatory drugs; PCS, phacoemulsification cataract surgery; POD1, first-day postoperative; SDD, same-day discharge; TASS, toxic anterior segment syndrome; UK, United Kingdom; US, United States

OpenAccess This article is distributed under the terms of the Creative Commons Attribution 4.0 International License (http:// creativecommons.org/licenses/by/4.0/), which permits unrestricted use, distribution, and reproduction in any medium, provided you give appropriate credit to the original author(s) and the source, provide a link to the Creative Commons license, and indicate if changes were made.

\section{References}

1. Kale MS (2011) “Top 5” lists top \$5 billion. Arch Intern Med 171: 1858

2. Grover M, McLemore R, Tilburt J (2016) Clinicians report difficulty limiting low-value services in daily practice. J Prim Care Community Health 7:135-138

3. Olson RJ, Braga-Mele R, Chen SH, Miller KM, Pineda R, Tweeten JP, Musch DC (2017) Cataract in the adult eye preferred practice pattern®. Ophthalmology 124:P1-P119

4. The Royal College of Ophthalmologists (2015) Commissioning guide: cataract surgery. https://www.rcophth.ac.uk/wp-content/ uploads/2015/03/Commissioning-Guide-Cataract-Surgery-FinalFebruary-2015.pdf. Accessed 20 Oct 2017

5. Lawrence D, Fedorowicz Z, van Zuuren EJ (2015) Day care versus in-patient surgery for age-related cataract. Cochrane Database Syst Rev 2:CD004242

6. Whitefield L, Crowston J, Little BC (1996) First day follow up for routine phacoemulsification? Br J Ophthalmol 80:148-150
7. Tufail A, Foss AJ, Hamilton AM (1995) Is the first day postoperative review necessary after cataract extraction? Br J Ophthalmol 79: 646-648

8. Zick J, Joondeph BC (2018) Is a postoperative day one examination needed after uncomplicated vitreoretinal surgery? Retina 38:331-333

9. Jaycock P, Johnston RL, Taylor H, Adams M, Tole DM, Galloway P, Canning C, Sparrow JM, UK EPR user group (2009) The cataract National Dataset electronic multi-centre audit of 55,567 operations: updating benchmark standards of care in the United Kingdom and internationally. Eye 23:38-49

10. Syed ZA, Moayedi J, Mohamedi M, Tashter J, Anthony T, Celiker C, Khazen G, Melki SA (2015) Cataract surgery outcomes at a UK independent sector treatment centre. Br J Ophthalmol 99:1460-1465

11. Greenberg PB, Tseng VL, Wu W-C, Liu J, Jiang L, Chen CK, Scott IU, Friedmann PD (2011) Prevalence and predictors of ocular complications associated with cataract surgery in United States veterans. Ophthalmology 118:507-514

12. McKellar MJ, Elder MJ (2001) The early complications of cataract surgery. Ophthalmology 108:930-935

13. Kohnen T, von Ehr M, Schütte E, Koch DD (1996) Evaluation of intraocular pressure with Healon and Healon GV in sutureless cataract surgery with foldable lens implantation. J Cataract Refract Surg 22:227-237

14. Rainer G, Menapace R, Findl O, Petternel V, Kiss B, Georgopoulos $M$ (2001) Effect of topical brimonidine on intraocular pressure after small incision cataract surgery. J Cataract Refract Surg 27:12271231

15. Unsal U, Baser G, Soyler M (2016) Intraocular lens implantation without the use of ophthalmic viscosurgical device. Int Ophthalmol $37: 25-30$

16. Jarstad JS, Jarstad AR, Chung GW, Tester RA, Day LE (2017) Immediate postoperative intraocular pressure adjustment reduces risk of cystoid macular edema after uncomplicated micro incision coaxial phacoemulsification cataract surgery. Korean J Ophthalmol 31:39-43

17. Bomer TG, Lagreze WD, Funk J (1995) Intraocular pressure rise after phacoemulsification with posterior chamber lens implantation: effect of prophylactic medication, wound closure, and surgeon's experience. Br J Ophthalmol 79:809-813

18. Elfersy AJ, Prinzi RA, Peracha ZH, Kim DD, Crandall DA, Darnley-Fisch DA, Imami NR (2016) IOP elevation after cataract surgery: results for residents and senior staff at Henry Ford Health System. J Glaucoma 25:802-806

19. Ahmed IIK, Kranemann C, Chipman M, Malam F (2002) Revisiting early postoperative follow-up after phacoemulsification. J Cataract Refract Surg 28:100-108

20. Slabaugh MA, Bojikian KD, Moore DB, Chen PP (2014) Risk factors for acute postoperative intraocular pressure elevation after phacoemulsification in glaucoma patients. J Cataract Refract Surg 40:538-544

21. Pohjalainen T, Vesti E, Uusitalo RJ, Laatikainen L (2001) Intraocular pressure after phacoemulsification and intraocular lens implantation in nonglaucomatous eyes with and without exfoliation. J Cataract Refract Surg 27:426-431

22. Cho YK (2008) Early intraocular pressure and anterior chamber depth changes after phacoemulsification and intraocular lens implantation in nonglaucomatous eyes. Comparison of groups stratified by axial length. J Cataract Refract Surg 34:1104-1109

23. Bonnell LN, SooHoo JR, Seibold LK, Lynch AM, Wagner BD, Davidson RS, Taravella MJ (2016) One-day postoperative intraocular pressure spikes after phacoemulsification cataract surgery in patients taking tamsulosin. J Cataract Refract Surg 42:1753-1758

24. Chang DF, Tan JJ, Tripodis Y (2011) Risk factors for steroid response among cataract patients. J Cataract Refract Surg 37:675-681 
25. Laurell CG, Wickström K, Zetterström C, Lundgren B (1997) Inflammatory response after endocapsular phacoemulsification or conventional extracapsular lens extraction in the rabbit eye. Acta Ophthalmol Scand 75:401-404

26. Kandarakis A, Soumplis V, Karampelas M, Panos C, Kyriakos N, Baxevanakis A, Karagiannis D (2010) Efficacy of brimonidine in preventing intraocular pressure spikes following phacoemulsification in glaucoma patients. Eur J Ophthalmol 20: 994-999

27. Levkovitch-Verbin H, Habot-Wilner Z, Burla N, Melamed S, Goldenfeld M, Bar-Sela SM, Sachs D (2008) Intraocular pressure elevation within the first 24 hours after cataract surgery in patients with glaucoma or exfoliation syndrome. Ophthalmology 115:104108

28. Takmaz T, Can I, Gürdal C, Kürkçüoğlu P, Așik S (2007) Effect of bimatoprost on intraocular pressure after phacoemulsification in eyes with exfoliation syndrome. Acta Ophthalmol Scand 85:262266

29. Desai P (1993) The national cataract surgery survey: II clinical outcomes. Eye 7:489-494

30. Zaidi FH, Corbett MC, Burton BJL, Bloom PA (2007) Raising the benchmark for the 21st century-the 1000 cataract operations audit and survey: outcomes, consultant-supervised training and sourcing NHS choice. Br J Ophthalmol 91:731-736

31. Clark A, Morlet N, Ng JQ, Preen DB, Semmens JB (2011) Whole population trends in complications of cataract surgery over 22 years in Western Australia. Ophthalmology 118:1055-1061

32. Theodore FH (1964) Corneal complications after cataract surgery. Int Ophthalmol Clin 4:913-948

33. Hsiao C-H, Ho C-H, Liao C-H, Wang H-Y, Wang J-J, Wu C-C (2014) Wound dehiscence as a cataract surgery-associated postoperative complication in patients previously treated with alpha-1 blocker tamsulosin - a population-based study in Taiwan. Am J Ophthalmol 158:1215-1220.e1

34. Chee S-P (2005) Clear corneal incision leakage after phacoemulsification-detection using povidone iodine $5 \%$. Int Ophthalmol 26:175-179

35. Shingleton BJ, Rosenberg RB, Teixeira R, O'Donoghue MW (2007) Evaluation of intraocular pressure in the immediate postoperative period after phacoemulsification. J Cataract Refract Surg 33: 1953-1957

36. Llop SM, Papaliodis GN (2018) Cataract surgery complications in uveitis patients: a review article. Semin Ophthalmol 33:64-69

37. Sengupta S, Chang DF, Gandhi R, Kenia H, Venkatesh R (2011) Incidence and long-term outcomes of toxic anterior segment syndrome at Aravind Eye Hospital. J Cataract Refract Surg 37: 1673-1678

38. Ozcelik ND, Eltutar K, Bilgin B (2010) Toxic anterior segment syndrome after uncomplicated cataract surgery. Eur J Ophthalmol 20:106-114

39. Perente I, Utine CA, Ozturker C, Cakir M, Kaya V, Eren H, Kapran Z, Yilmaz OF (2007) Evaluation of macular changes after uncomplicated phacoemulsification surgery by optical coherence tomography. Curr Eye Res 32:241-247

40. Mamalis N (2013) Intraocular lens dislocation. J Cataract Refract Surg 39:973-974

41. Ascaso FJ, Huerva V, Grzybowski A (2015) Epidemiology, etiology, and prevention of late IOL-capsular bag complex dislocation: review of the literature. J Ophthalmol 2015:805706

42. Mamalis N, Brubaker J, Davis D, Espandar L, Werner L (2008) Complications of foldable intraocular lenses requiring explantation or secondary intervention - 2007 survey update. J Cataract Refract Surg 34:1584-1591

43. Vazquez-Ferreiro P, Carrera-Hueso FJ, Fikri-Benbrahim N, Barreiro-Rodriguez L, Diaz-Rey M, Barrios MAR (2016) Intraocular lens dislocation in pseudoexfoliation: a systematic review and meta-analysis. Acta Ophthalmol 95:e164-e169

44. Fernández-Buenaga R, Alió JL (2017) Intraocular lens explantation after cataract surgery: indications, results, and explantation techniques. Asia Pac J Ophthalmol (Phila) 6:372-380

45. Chakrabarti A, Nazm N (2017) Posterior capsular rent: prevention and management. Indian J Ophthalmol 65:1359-1369

46. Kessel L, Andresen J, Erngaard D, Flesner P, Tendal B, Hjortdal J (2015) Safety of deferring review after uneventful cataract surgery until 2 weeks postoperatively. J Cataract Refract Surg 41:27552764

47. Tan P, Foo FY, Teoh SC, Wong HT (2014) Evaluation of the use of a nurse-administered telephone questionnaire for post-operative cataract surgery review. Int J Health Care Qual Assur 27:347-354

48. Allan BDS, Baer RM, Heyworth P, Duguid IGM, Dart JKG (1997) Conventional routine clinical review may not be necessary after uncomplicated phacoemulsification. Br J Ophthalmol 81:548-550

49. Eloranta H, Falck A (2017) Is an ophthalmic check-up needed after uneventful cataract surgery? A large retrospective comparative cohort study of Finnish patients. Acta Ophthalmol 95:665-670. https://doi.org/10.1111/aos.13373

50. Ibrahim N, Pozo-Martin F, Gilbert C (2015) Direct non-medical costs double the total direct costs to patients undergoing cataract surgery in Zamfara state, Northern Nigeria: a case series. BMC Health Serv Res 15:163 